\title{
Seeking transparency
}

\author{
A more transparent drug approval process has the potential to reduce cost and duplicated effort, as well as \\ ultimately improve access to effective treatments for patients.
}

$\mathbf{T}^{\mathrm{h}}$ he US Food and Drug Administration (FDA), generally a tightlipped agency, could be set for a change. The agency is proposing to release information that is currently kept behind a wall, such as when an application for a new drug has been submitted or denied. If implemented, some of the 21 measures proposed by the FDA would represent a major change in how the agency does business-and could change not only how drugs are prescribed but also how companies and research institutions design clinical trials and preclinical studies. Backing from the biomedical research community could help make the proposals a reality.

Shortly after the handover of the FDA to the Obama administration, the newly appointed principal deputy commissioner Joshua Sharfstein launched a task force to increase transparency (Nat. Med. 16, 244, 2010). The 21 new draft proposals emerge as part of this initiative. They range from measures to increase public access to adverse event reports to the status of inspections of drug manufacturers.

Particularly relevant for the research community are several proposals that would make available information about drug company research that is currently kept confidential. The FDA would, for instance, disclose when a company files an Investigational New Drug Application (IND), thereby initiating clinical trials, and whether the application was denied, put on hold or approved. The agency would also disclose when a company submits an application to market a new drug or an old drug for a new indication-and provide the public with the letter of response it provides the sponsor, outlining its reasons if the application is rejected. The agency could also disclose summary safety and effectiveness data from an IND or marketing application if it concludes that the release of such information would serve the interests of the public health.

One aim of the new proposals is to accelerate innovation and reduce duplication of efforts by enabling drug developers to learn from the outcome of FDA applications. For instance, one proposed rule would open the lid on information about orphan drugs, publicizing whether an application was abandoned by the sponsor for reasons unrelated to safety and whether the FDA thinks the drug has the potential to become a major therapeutic advance. The new rules would open the door to new sponsors, such as patient groups, to take up development of abandoned drugs.

The proposals could also reduce the huge public health problem of the overprescription of already approved drugs. Take atypical antipsychotics such as Seroquel. These drugs, which can have severe side effects on bone and weight, are often prescribed off label for conditions such as attention deficit hyperactivity disorder. If a drug company were to initiate a trial to test whether the drugs work for this condition, the public would know-and they would know the FDA's stance on the results. Such information is potentially powerful-even more so if the agency could provide it retroactively.

It is not yet certain that proposals will be implemented. The agency walks a fine line between its mandate to protect public health and its obligation to protect trade secrets. Some industry representatives have gone on record saying that they prefer the status quo, maintaining that increased transparency would quash innovation. For some of the proposals, that is a fair question to ask-how would alerting potential competitors to a new IND affect incentives for drug development? The FDA considered such issues, for instance calling for redaction of information related to how a drug is made. Further discussions will help forge reasonable compromises.

Other complaints about incentive quashing don't hold up to close scrutiny. For instance, the European Medicines Agency already discloses its evaluation of a drug if a marketing application is withdrawn, and most drug developers now operate globally. Moreover, a freer flow of information has the potential to level the playing field for all drug developers.

Researchers who have done the difficult work of mining existing FDA databases for unpublished clinical trial data have said they would like the FDA to be even more open. To that end, the FDA is proposing further discussion about the release of more comprehensive safety and effectiveness data, beyond the summary information covered by the current proposals.

In the end, new rules and regulations mean little if they are not implemented fully. The FDA, for instance, has yet to fully hew to its long-standing policy of releasing its analyses of drugs approved for new indications. Moreover, clinical trial sponsors are already required to register their trials with a US National Institutes of Health (NIH) database, but a recent analysis showed that only about half do so in a timely and complete way (J. Am. Med. Assoc. 302, 977-984, 2009). The new FDA proposals could synergize with the NIH effort and provide context to the data. But, ideally, clinical trial registration and release of FDA information about those trials would proceed in a coordinated way.

In the balance, a more open FDA would strengthen the research enterprise and serve the interests of public health. Increased transparency would also help forge a culture change at the FDA that could outlast the current administration, making the agency more accountable to the public.

We urge our readers to weigh in to the FDA with their thoughts, before July 20, when the period for public comment ends. Readers can access the proposals here (http://fdatransparencyblog.fda.gov/2010/05/ fda-unveils-draft-proposals-on-agency-disclosure-policies-for-publiccomment.html/). The new FDA is listening. 